\title{
BMJ Quality Developing Quality Improvement capacity and capability across the Children in Fife partnership
}

\author{
Craig Morris, Ingrid Alexander
}

To cite: Morris C, Alexander I. Developing Quality Improvement capacity and capability across the Children in Fife partnership. BMJ Quality Improvement Reports 2016;5:u212664. w5045. doi:10.1136/ bmjquality.u212664.w5045

Received 22 June 2016 Revised 19 August 2016

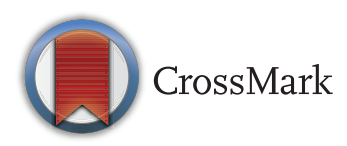

Scotland, United Kingdom

Correspondence to Craig Morris craig.morris@fife.gov.uk

\section{ABSTRACT}

A Project Manager from the Fife Early Years Collaborative facilitated a large-scale Quality Improvement (herein QI) project to build organisational capacity and capability across the Children in Fife partnership through three separate, eight month training cohorts.

This 18 month QI project enabled 32 practitioners to increase their skills, knowledge, and experiences in a variety of $\mathrm{Q}$ l tools including the Model for Improvement which then supported the delivery of high quality improvement projects and improved outcomes for children and families. Essentially growing the confidence and capability of practitioners to deliver sustainable QI.

27 respective improvement projects were delivered, some leading to service redesign, reduced waiting times, increased uptake of health entitlements, and improved accessibility to front-line health services. 13 improvement projects spread or scaled beyond the initial site and informal QI mentoring took place with peers in respective agencies. Multiple PDSA cycles were conducted testing the most efficient and effective support mechanisms during and post training, maintaining regular contact, and utilising social media to share progress and achievements.

\section{PROBLEM}

Fife Community Planning Partnership has five (May 2016) local and national QI programmes that span a child's journey from conception to early adulthood. All of these programmes use the same improvement methodologies in an attempt to improve outcomes for children and families.

In September 2014, one of these programmes, namely the Fife Early Years Collaborative (EYC) pinpointed a need to recruit, train, and support practitioners to deliver sustainable change projects. Approximately 60 improvement activities were recorded at this time. However, it was recognised that people across the partnership had only been trained to an introductory level of QI and lacked the skills and knowledge to successfully spread and sustain improvements which were demonstrating impact. The QI project sought to record the following aims statement, 30 Fife EYC practitioners be trained and confident in using the Model for Improvement and other QI tools to deliver sustainable change projects by April 2016. This was refined in September 2015, from 20 to 30 and from December 2015 to April 2016.

With more practitioners becoming aware of these improvement programmes, and a change of culture resulting in more practitioners being encouraged to lead change and improvement, the challenge was to support and subsequently sustain improvements by building organisational QI capability.

A theory and hunch was that by empowering, training, and supporting practitioners to an advanced level, a level between intermediate and lead level improvement, this would further embed and sustain QI cultures across the Children in Fife partnership. These individuals would then become the 'change agents' in their respective arena to provide organisational support and sustainability.

\section{BACKGROUND}

Transformational change requires leaders who are trained to direct the effort and there are few empirical examples of programmes that seek to develop QI leaders. ${ }^{1}$ Furthermore, encouraging individuals to implement and test through small scale testing can be more successful than facilitating single comprehensive interventions. ${ }^{1}$

Predominately QI training of Healthcare staff $^{2}$ is offered from undergraduate to postgraduate levels and within specific clinical professional groups. Clinical outcomes can improve through training leaders in QI tools and coaching. A multi-professional approach to educating and delivering QI is required, to enable healthcare staff to view improvement as an organisational, professional, and 
clinical issue. ${ }^{2}$ The development of safe and effective services requires strong leadership and improvement in the 'micro-system' occurs when practitioners work and where there can be real gains in the quality, value, and safety of front-line delivery. ${ }^{3}$

For a truly post-Taylorism health workforce to prevail, it would have to be one that has the skillbase to set aims, measure progress, test new ways of working, and are empowered to make bold changes to change the system. ${ }^{4}$ Similarly, this an excellent time to invest in the workforce that is imaginative, inspired, and capable to empower thinking to reinvent the system. Moreover, medical and healthcare training does not necessarily prepare practitioners for the every day environment where continual improvement is required. ${ }^{5}$ If frontline practitioners are given the right tools and knowledge to nurture leaderships at all levels, and given support to understand complex systems this will bring about real change and improvement. ${ }^{6}$ Health professionals face pressures to improve quality and measurable outcomes without having systems in place that can help them easily identify the best practices for a given case or means of arranging for follow-up on a patient's needs across the entire continuum of care. ${ }^{7}$

In Scotland there are a number of QI programmes, for leaders in the public sector, namely the Scottish Improvement Leaders programme (cohort 1 August 2014) and the Clinical Fellowship programme (at June 2016 cohort 9). There are Masters level QI qualifications at various universities across the United Kingdom, with 37 accredited post-graduate courses (MSc \& Diploma) on the Universitites and Colleges Admissions Services (UCAS) database. The field of improving quality is also broad and open to broad interpretation. ${ }^{8}$

\section{BASELINE MEASUREMENT}

At the start of the improvement project a measure of the cumulative number of QI Projects being delivered within the Children in Fife partnership was taken. This measurement enabled the project team to determine the overall impact of projects being delivered reference each of the three cohorts across the Children in Fife partnership.

A second measure (Figure 1) was using the IHI project scale to demonstrate the progress of each project on a recognised and robust basis. As this was introduced at the start of the improvement project, this inevitably indicated a zero starting point. Measured on a monthly basis this provided an opportunity to demonstrate if the increased skills and knowledge resulted in more projects scaling and spreading.

The third measurement was practitioner confidence levels before, during and after the programme. This was a scaling of confidence (1-5) on knowledge and skills of application of project tools, project delivery, and mentoring peers.

Other process measures which formed a project dashboard included attendance, completion of IHI Open School, monthly project returns received, use of Social Media to share project progress, and individual achievements, Project Manager hours supporting practitioner and requests for support.

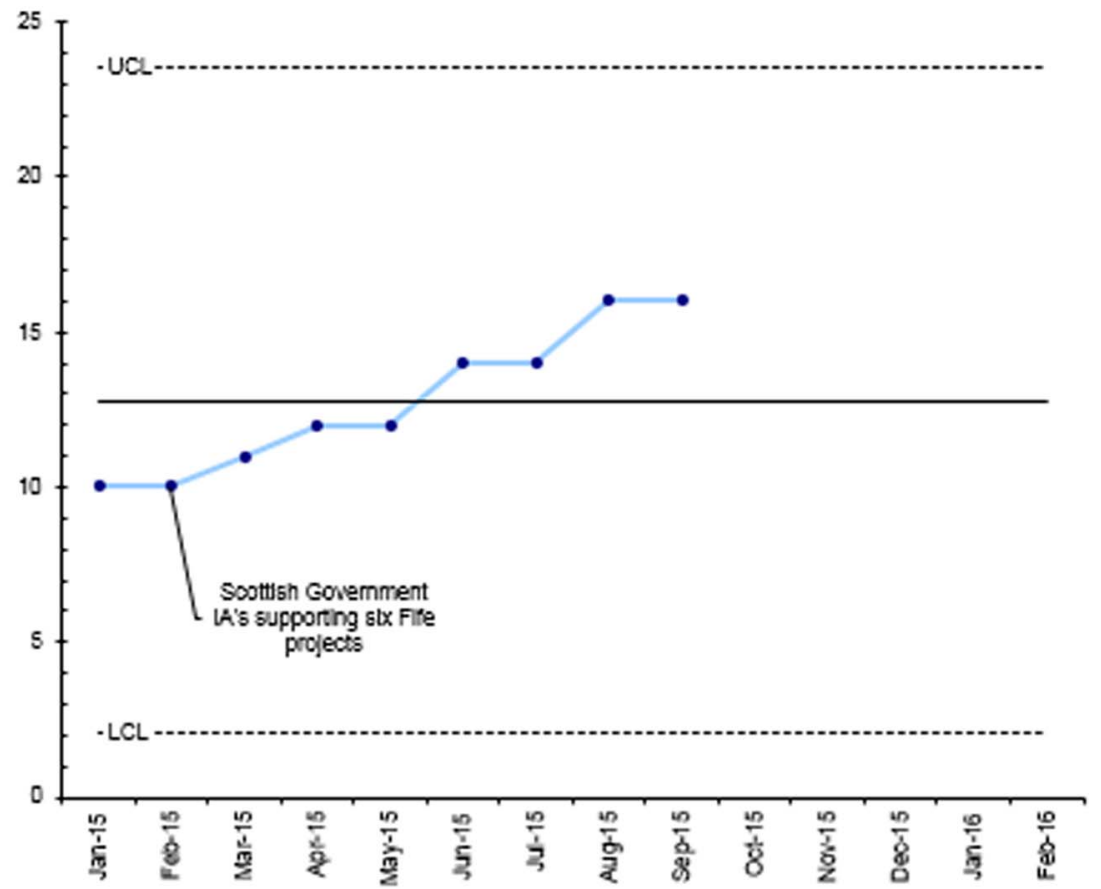




\section{DESIGN}

Three eight month training programmes were delivered between October 2014 \& April 2016. A multi-agency team which comprised, Fife Early Years Collaborative Project Manager, Improvement Programmes Manager (NHS Fife), Fife Council Team Manager (IHI Improvement Advisor Wave 37), NHS Organisational Development Consultants, and an Improvement Advisor from the Scottish Government Early Years Collaborative national team all supported this Workforce Development programme.

Cohort 1 comprised of 9 and ran from August 2014 to February 2015. Cohort 2 had 19 and ran from April 2015 to December 2015. Cohort 3 had 4 and ran from September 2015 to April 2016. Cohort 2 had 25 individuals starting the programme but only 19 completed. For cohort 2, practitioners and their line managers completed the programme jointly. The programme was open for all to attend on a first come first served basis.

Cohort 1 and 2 had an equal focus towards human factors balanced with the technical skills and knowledge to deliver change. Cohort 3 only focussed on technical QI knowledge and skills. The training was predominately delivered through one day a month for eight months with mentoring surgeries facilitated between each session. Prior to the course participants were expected to complete a short work pack. On completion of each training block participants were expected to embed QI learning into practice through delivering a QI project. Participants took on self-directed courses through the IHI Open School.

Nine separate workshops were delivered over an eight month period for three cohorts. The first block was run over two days to front-load technical improvement knowledge in order that identification of improvement projects. This included Kotter's model of change, extensive depth of improvement planning toolkits, and stakeholder analysis to enable practitioners to identify and plan for their improvement project. Practitioners were introduced to monthly reporting and supported to complete an improvement project charter. Workshop three and four supported aims setting, further knowledge on the Model for Improvement, and driver diagrams followed by data and measurement. By workshop four practitioners were expected to have completed specific modules from the IHI Open School related to the programme delivery.

At every meeting significant time was built in to refine project charters and review respective progress. The Human Side of change which included coaching discussions started at workshop five and six. Workshop seven discussed the critical considerations for spreading and scaling successful improvement work. The final two workshops supported practitioners to teach Quality Improvement within their organisations and the facilitation skills needed to do this. Senior Managers of the practitioners were invited, along with other interested stakeholders, to the final session to celebrate the progress of each cohort. Practitioners were encouraged to showcase their improvement project in the form of a storyboard. This was then shared by organisational communication to celebrate the impact of the improvement programme completed.

\section{STRATEGY}

Multiple and iterative PDSA cycles were facilitated, specifically around maintaining contact with practitioners, ensuring bespoke QI support was provided, maintaining high attendance at workshops, and use of social media to share progress in respective practitioner learning and QI projects.

Several PDSA cycles (Supplementary material 1 Providing bespoke to practitioners outwith training sessions) were conducted to establish what would work best and most efficiently for practitioners attending the eighth-month training programme. It was predicated that over $80 \%$ of practitioners would attend the monthly surgeries. Interestingly though quick and weekly PDSA cycles, it was established the most popular and articulated support mechanism from practitioners was not the vehicle which worked most efficiently in practice. Two cohorts specifically asked for coaching surgeries outwith the sessions at a central venue, based on measuring attendance and quality feedback from support sessions. When hosting a number of these surgeries this resulted in little or no attendance. Testing took place to increase the numbers attending support appointments, such as changing the days, location, and times of these workshops. After five PDSA cycles, it was established and implemented the most efficient support was through 30 minute phone check ins. This was in line with the reducing capacity of frontline practitioners to host through ever increasing case loads and other competiting pressures. It also meant travel time was reduced for both the facilitator and the practitioner.

Practitioners on the programme were asked to submit monthly updates to detail the progress within their improvement projects. This would enable the support team to direct appropriate support. At the start, the project team believed that there would be a lower than $30 \%$ return rate given the challenges around front-line capacity. After one month, there was approximately $40 \%$ returns. Through speaking with a practitioner who did return they advised that the word 'return' provides negative connations and should be changed to 'update'. The project team predicted this would result in little or no difference for PDSA cycle 2. On the second cycle the word changed from 'return' to 'update' and monthly returns increased to $70 \%$ of those attending. PDSA Cycle 3 sought to retain the word 'update' and send a gentle reminder by email the week previous to these being scheduled. This generated the same level of returns. It was found that on cohort 2 , those who were not regularly returning monthly updates after month four, did not complete the eight month programme. 
Use of social media to share learning from practitioner improvement projects. This test was abandoned after three PDSA cycles as it was found a low number of practitioners attending cohort one and two either used or had an appetite to use Social Media to share their learning.

\section{RESULTS}

Increase in the number of QI projects spreading to more than one delivery site:

In total 13 projects spread to more than one site \& three mainstreamed within eight months of respective cohort finishing (May 16). Three have been sustained as everyday business. This included reduction in waiting times to one service, increase in the uptake of health entitlements across Fife, increased numbers attending health appointments, increase in parental confidence, and a significant reduction in avoidable rescreening of patients in one service. Three QI projects were abandoned within six months of inception and one project did not transition into testing stage.

By measuring the cumulative number of projects and introducing the IHI Project Scale Assessment Framework ensured more robust QI projects were being facilitated (Figure 2). This resulted in the number of QI projects reducing as the better operational definition of a QI project. It was difficult to measure on a monthly basis as required the goodwill of practitioners to return information on the progress of respective projects. From time QI project started to time finished there was a substantial reduction in projects reported, however, more projects spreading and scaling than when starting. Essentially demonstrating that increased knowledge to spread projects was emerging from the three cohorts delivered.

QI learning is a journey and not a destination: Furthering professional development in areas of QI by practitioners attending. One individual transitioned to IHI Improvement Advisor course. 15 practitioners completed first level of IHI Open School. Other opportunities to consolidate the learning on the programme, e.g. Run Chart building was offered to the 27 practitioners. Eight practitioners on this programme also engaged in opportunities to 'brush up' their learning, post completion, in particular areas by attending introductory bitesize QI training workshops.

Embedding QI cultures within organisations: Two senior practitioners developed a learning culture for QI in their respective agencies and hosted monthly 'Test of Change' meetings. Informal peer mentoring in the delivery of QI work. 33\% ( $n=12)$ practitioners from cohort one commented that returning to base to embed the learning was difficult as knowledge levels of colleagues and managers was non-existent to provide ongoing support. Subsequently cohort two, requested managers and practitioners to complete the programme jointly.

Cross cultural learning, 11 different agencies across the Children In Fife partnership attended the programme. Feedback from practitioners told that cross cultural learning was helpful to progress improvement and share learning across different cultures.

High numbers starting and finishing the programme. $84 \%(\mathrm{n}=32)$ of practitioners starting the programme completed the eight month training, 92\% ( $\mathrm{n}=27)$ had a $90 \%$ attendance or greater across nine workshops. 27 practitioners delivered a respective improvement project

Figure 2

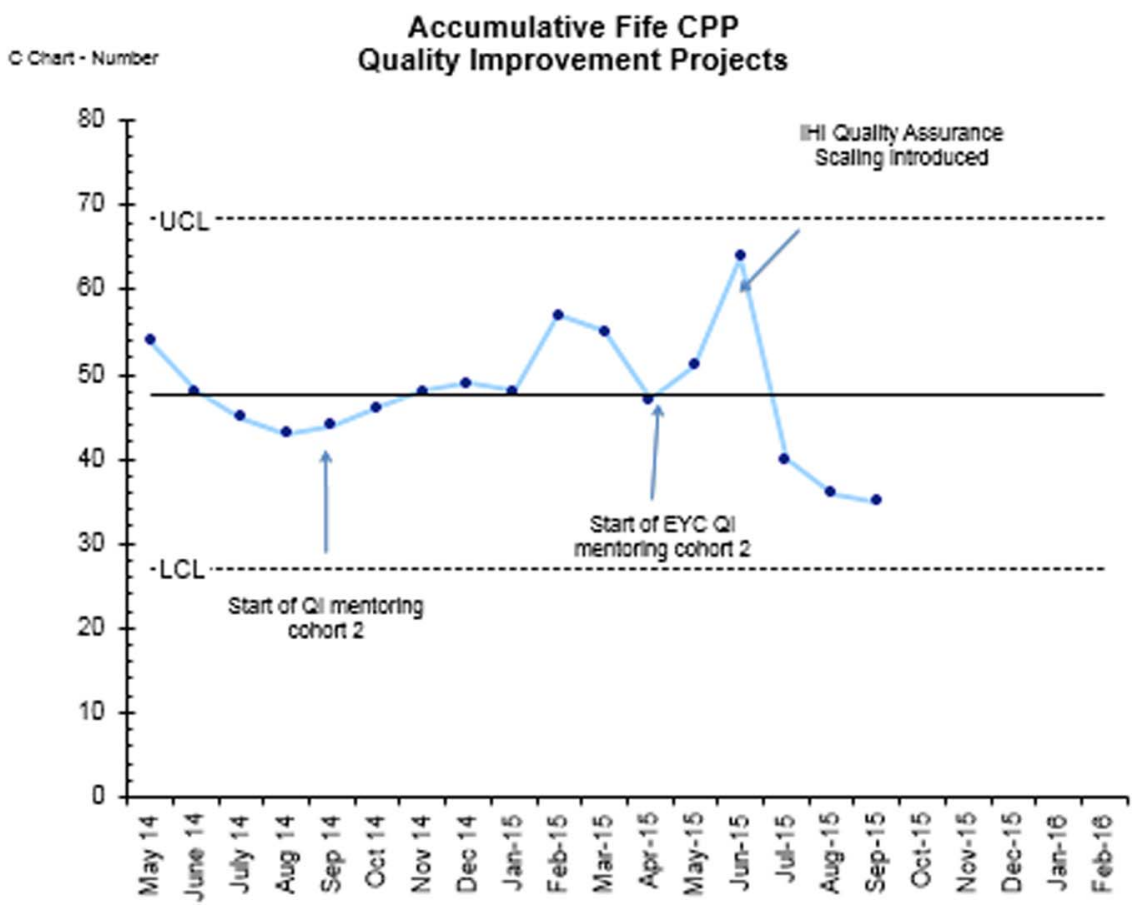


that could lead to service improvement and subsequently improve outcomes for children and families.

Practitioners informally mentoring their peers in various areas of Quality Improvement. All practitioners in cohort one or two $(n=23)$ informed that they had carried out QI Mentoring in their respective organisations, on an ad-hoc needs-led basis. Confidence levels increased for $62 \%(n=27)$ of participants, by a scale of 2 points (1 lowest - 5 highest) for areas of QI mentoring, application of tools and project execution. These results are only applicable to practitioners on cohort $1 \& 2$.

\section{LESSONS AND LIMITATIONS}

The numbers of practitioners trained through this programme as part of the Children in Fife partnership are a small percentage to evoke change and improvement at large scale. This QI project was a multi-agency collaboration and a small part of facilitators work plans. To effectively continue more resource and capacity would be needed to deliver at the large scale.

A number of PDSA cycles were conducted to ascertain most efficient and effective support outwith the programme. 30 minute support check-ins by phone once a month were implemented and sustained high contact numbers throughout cohort 2 and 3 . This in cognizance of facilitators and practitioners workload pressures, as it proved challenging to allocate appropriate time to complete learning tasks and deliver QI projects outwith the training.

Prior to the programme starting, an aim was to measure the number of monthly mentoring appointments each practitioner would host after completing the eight month programme. A three month post post programme follow up meeting with all cohorts was hosted to determine any challenges and detail progress to embedding QI learning. All individuals in cohort one and two $(n=23)$ respectively had facilitated mentoring and coaching discussions in areas of QI within their organisations, however, this was more ad-hoc than respective structured support planned. Two practitioners (cohort one \& two) had introduced monthly 'Test of change' meetings to harness QI work in respective organisations.

PDSA learning and other feedback received was critical to using accessible language in programme deliveries and follow up support. Key words in the programme were changed to align with practice, plain english principles, and practitioner understanding. Cohort one informed unanimously that their expectations of coaching did not match what was delivered in practice. They recommended the term 'Mentoring', than 'Coaching' for cohort two. As the later has connations with qualifications, progressive levels, and formality. Monthly reporting was changed to monthly updates.

Higher than predicted numbers of practitioners completed the programme moved professional roles from when the programme started. A course aim was to build organisational capacity to execute and sustain QI.
Naturally this knowledge and skill set transfers to new position but organisationally the capacity and capability to support practitioners will inevitably be reduced. From a facilitator perspective $50 \% \quad(n=6)$ of those facilitating the programme have moved positions since time of starting cohort one. Cohort one, $54 \%$ practitioners $(n=12)$, cohort two $12.5 \%(n=16)$, and cohort three $50 \%$ of practitioners $(n=4)$ had moved position from the time they started the programme or taken a temporary period of absence as recorded at May 2016.

\section{CONCLUSION}

Mid way through the QI project the aims statement was refined from 20 to 30 individuals, and the completion date moved forward six months. This was in cognizance of the higher than predicated interest and completion of programme in cohort one and two. Accordingly resulting an additional cohort (three) being delivered.

Programme costs were minimal as professional time was given in-kind and required multi-agency commitment to ensure each cohort was delivered and met its objectives. Going forward significant resource investment and strategic leadership commitment to this programme or similar would be needed to deliver and sustain at a larger level. The programme received local and national attention reference results recorded but the sustainability to maintain the level of deliveries from a facilitator perspective and practitioners attending one day a month, will prove challenging in times of unprecedented change in local government and the NHS.

Overall, $27 \%$ of practitioners and $50 \%$ of facilitators who engaged or delivered the programme have moved posts from when they started the programme, This inevitably will have an impact on the ability for organisations to embed and sustain QI cultures and the Children In Fife partnership to maintain continuity in future training deliveries.

High numbers attended all three programme cohorts, $84 \%$ of practitioners who started went on to complete the programme and $48 \%$ of improvement projects scaled and spread to a second site. Underpinning that increased knowledge and confidence in all areas of QI is an enabler to achieving more robust and sustainable change and improvement.

Despite a significant proportion of staff and facilitator turnover, the skills and knowledge gained are applicable to any environment. The training programme design can be quickly replicated and offered more widely to the Children In Fife partnership, if respective multi-agency investment and commitment is secured.

Acknowledgements Ingrid Alexander - Improvement Programmes Manager, NHS Fife for supporting the programme from inception to completion. Furthermore for their enthusiasm, support and coaching as Scottish Improvement Leaders Programme organisational mentor.

Contributors to the programme:

Sacha Will - Scottish Government Improvement Advisor, Early Years Collaborative.

Mairi Ferris - Fife Council (Improvement Advisor - IHI wave 37) Nicola Harkins \& Joanne Rafferty - NHS Flfe, Organisational Development 
To all participants who subscribed to the programmme and applied the skills and knowledge to everyday practice, the improvements are tangible and recorded positive outcomes for children and families across Fife.

Leadership and enthusiasm Fife Partnership, for permission and support to embedding a QI culture across the Children In Fife partnership as 'everyday business'.

Declaration of interests Nothing to declare.

Ethical approval No ethical approval was sought for this project. All practitioners participated on the programme by voluntary accord and any respective improvement project was determined by those attending. The improvement projects were primarily intended to improve local healthcare. No practitioner should be identifiable through any part of the article.

Open Access This is an open-access article distributed under the terms of the Creative Commons Attribution Non-commercial License, which permits use, distribution, and reproduction in any medium, provided the original work is properly cited, the use is non commercial and is otherwise in compliance with the license. See:

- http://creativecommons.org/licenses/by-nc/2.0/

- http://creativecommons.org/licenses/by-nc/2.0/legalcode

\section{REFERENCES}

1. G Kaminski, MT Britto et al. Developing capable quality improvement leaders. BMJ Quality Safe 2012;21:903-11

2. S Heard, G Schiller et al. Continuous quality improvement; education towards a culture of clinical governance. Quality in Health Care 2001;10:ii70;ii78

3. J Mohr, P Batalden. Improving safety on the front line; the role of clinical microsystems. Quality Safety in Health Care 2002:11:45-50

4. D M Berwick. Improvement, trust, and the healthcare workforce. Qual Safe Health Care 2003;12:i2-i6 Downloaded from http:// qualitysafety.bmj.com/ on June 2, 2016

5. J Runnacles, A Moult et al. Developing future clinical leaders for quality improvement: experience from a London children's hospital. FMJ Qual Safety 2013;22:956-63

6. A Roueche, j Hewitt. 'Wading through treacle'; quality improvement lessons from the frontline. BMJ Quality Safety 2012;21:179-83

7. Committee on Quality of Health Care in America. Crossing the Quality Chasm. A new Healthcare for the 21st century. Washington, DC, USA: Institute of Medicine - National Academies Press. 2001.

8. UCAS. https://digital.ucas.com/courses/details?coursePrimaryld= 18f83b53-8708-ef8c-b098-e8ae127f9ef9\&courseOptionld= d43a9152-7bf9-f9fe-88a6-4098238c7151. Year of publication 2016. (Accessed 21 May 2016) 